\title{
Shock Breakout of Type II Plateau Supernova
}

\author{
Nozomu Tominaga ${ }^{1,2}$, Tomoki Morokuma ${ }^{3}$ and Sergei I. Blinnikov ${ }^{4,2}$ \\ ${ }^{1}$ Department of Physics, Faculty of Science and Engineering, Konan University, \\ 8-9-1 Okamoto, Kobe, Hyogo 658-8501, Japan \\ email: tominaga@konan-u.ac.jp \\ ${ }^{2}$ Kavli Institute for the Physics and Mathematics of the Universe, University of Tokyo, \\ 5-1-5 Kashiwanoha, Kashiwa, Chiba 277-8583, Japan \\ ${ }^{3}$ Institute of Astronomy, University of Tokyo, Mitaka, Tokyo 181-0015, Japan \\ ${ }^{4}$ Institute for Theoretical and Experimental Physics (ITEP), Moscow 117218, Russia
}

\begin{abstract}
Type II-plateau supernovae (SNe II-P) are fainter than Type Ia SNe and thus have so far been observed only at $z<1$. We introduce shock breakout and propose a distant SN II-P survey at $z>1$ with shock breakout. The first observation of shock breakout from the rising phase is reported in 2008. We first construct a theoretical model reproducing the UV-optical light curves (LCs) of the first example and demonstrate that the peak apparent $g$-band magnitude of the shock breakout would be $m_{\mathrm{g}} \sim 26.4$ mag if an identical SN occurs at a redshift $z=1$, which can be reached by $8 \mathrm{~m}$-class telescopes. Furthermore, we present LCs of shock breakout of SN explosions with various main-sequence masses, metallicities, and explosion energies and derive the observable SN rate and reachable redshift as functions of filter and limiting magnitude by taking into account an initial mass function, cosmic star formation history, intergalactic absorption, and host galaxy extinction. The $g$-band observable SN rate with limiting magnitude $27.5 \mathrm{mag}$ is $3.3 \mathrm{SNe} \mathrm{deg}^{-2}$ day $^{-1}$ and half of them are located at $z>1.2$.
\end{abstract}

Keywords. shock waves, radiative transfer, supernovae: general, supernovae: individual (SNLS04D2dc), stars: evolution, surveys

\section{Introduction}

Shock breakout is the bolometrically brightest phenomenon in supernovae ( $\mathrm{SNe}$ ) and thus it is suggested to be observable even if it takes place at $z \gtrsim 1$ (e.g. Chugai et al. 2000). Recently, shock breakout of Type II plateau SNe (SNe II-P) was detected for SNLS-04D2dc (Schawinski et al. 2008; Gezari et al. 2008) and SNLS-06D1jd (Gezari et al. 2008) by the GALEX satellite and thus it is coming under the spotlight to probe high- $z$ CCSNe by the shock breakout of SNe II-P. However, the observable properties are poorly understood. Therefore, in this contribution, we present theoretical predictions of observables of shock breakout of SNe II-P calculated by a multigroup radiation hydrodynamics code STELlA (Blinnikov et al. 1998; 2000; 2006).

\section{Results}

First, we focus on SNLS-04D2dc because SNLS-06D1jd has sparse UV observations with relatively low signal-to-noise ratio and compare the synthetic LCs with the multicolor observations of SN IIP SNLS-04D2dc. We successfully construct a SN II-P model reproducing well the shock breakout and plateau consistently and constrain SN and progenitor properties (left panel of Fig. 1, Tominaga et al. 2009). Furthermore, based on the multicolor LC model, we demonstrate that the peak apparent $g$-band magnitude of the 

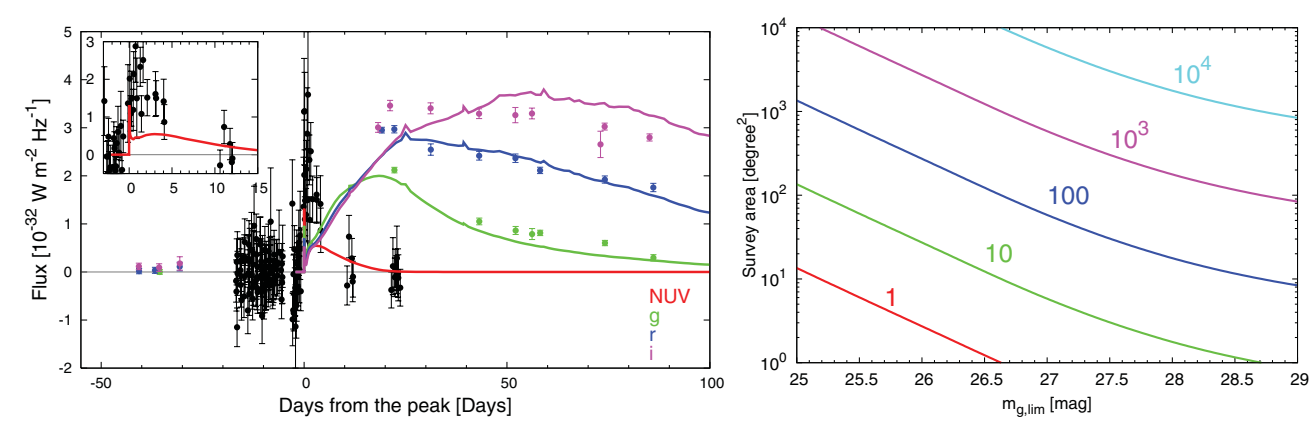

Figure 1. (Left)Comparison between the SNLS-04D2dc observations (points) and a SN IIP model reddened for the host galaxy extinction (lines) (black and red: near UV, green: $g$ band, blue: $r$ band, magenta: $i$ band). The inset enlarges the phase when the SN emitted UV light. (Right) Observable SN rate per day in $g^{\prime}$ band. The lines show different observable SN rate: 1 (red), 10 (green), 100 (blue), $10^{3}$ (magenta), and $10^{4}$ (cyan).

shock breakout would be $m_{\mathrm{g}} \sim 26.4 \mathrm{mag}$ if a SN being identical to SNLS-04D2dc occurs at a redshift $z=1$, which can be reached by $8 \mathrm{~m}$-class telescopes. The result evidences that the shock breakout has a great potential to detect SNe IIP at $z \gtrsim 1$.

Next, we adopt progenitor models with various main-sequence masses which are taken from Umeda \& Nomoto (2005) and present multicolor light curves of shock breakout of SNe II-P (Tominaga et al. 2011). As a result, we predict apparent multicolor light curves of shock breakout at various redshifts $z$ and derive the observable SN rate and reachable redshift as functions of filter $x$ and limiting magnitude $m_{x, \text { lim }}$ by taking into account an initial mass function, cosmic star formation history, intergalactic absorption, and host galaxy extinction. We propose a realistic survey strategy optimized for shock breakout. For example, the $g^{\prime}$-band observable SN rate for $m_{g^{\prime}, \text { lim }}=27.5 \mathrm{mag}$ is 3.3 SNe degree ${ }^{-2}$ day $^{-1}$ and half of them are located at $z \geqslant 1.2$ (right panel of Fig. 1).

Although the survey parameters should be customized to observation purposes and telescope/instrument, future/ongoing wide and/or deep surveys, e.g. Palomar Transient Factory (PTF), Lick Observatory Supernova Search (LOSS), Catalina Real-Time Transient Survey (CRTS), Kiso/Kiso Wide Field Camera (KWFC), Skymapper, Dark Energy Survey (DES), Panoramic Survey Telescope and Rapid Response System (Pan-STARRS), Subaru/Hyper Suprime-Cam (HSC), and Large Synoptic Survey Telescope (LSST), will find a large number of shock breakout events. We emphasize that the multicolor observations in blue optical bands with $\sim$ hour intervals, preferably over $\geqslant 2$ continuous nights, are essential to efficiently detect, identify, and interpret shock breakout and conclude that the most essential observation is the multicolor photometry with short intervals less than 1 day and that the observation over $\geqslant 2$ continuous nights is favorable.

\section{References}

Blinnikov, S., Lundqvist, P., Bartunov, O., Nomoto, K., \& Iwamoto, K. 2000, ApJ, 532, 1132

Blinnikov, S. I., Eastman, R., Bartunov, O. S., et al., 1998, ApJ, 496, 454

Blinnikov, S. I., Röpke, F. K., Sorokina, E. I., et al., 2006, A\& A, 453, 229

Chugai, N. N., Blinnikov, S. I., \& Lundqvist, P. 2000, Mem. della Soc. Astro. Ita., 71, 383

Gezari, S., Dessart, L., Basa, S., et al. 2008, ApJL, 683, L131

Schawinski, K., Justham, S., Wolf, C., et al. 2008, Science, 321, 223

Tominaga, N., Blinnikov, S., Baklanov, P., et al., 2009, ApJL, 705, L10

Tominaga, N., Morokuma, T., Blinnikov, S., et al., 2011, ApJS, 193, 20

Umeda, H. \& Nomoto, K. 2005, ApJ, 619, 427 